\title{
The developmental transcriptome of the synanthropic fly Chrysomya megacephala and insights into olfactory proteins
}

\author{
Xiaoyun Wang, Mei Xiong, Chaoliang Lei and Fen Zhu*
}

\begin{abstract}
Background: Chrysomya megacephala (Fabricius) is a prevalent and synanthropic blowfly which has two sides, for being a pathogenic vector, an efficient pollinator, a promising resource of proteins, lipids, chitosan, biofuel et al., and an important forensic indicator. Moreover olfactory proteins are crucial component to function in related processes. However, the genomic platform of C. megacephala remains relatively unavailable. Developmental transcriptomes of eggs, larvae from 1st instar to before pupa stage and adults from emergence to egg laying period were built by RNA-sequencing to establish sequence background of $C$. megacephala with special lights on olfactory proteins.
\end{abstract}

Results: Clean reads in eggs, larvae and adults were annotated into 59486 transcripts. Transcripts were assembled into 22286, 17180, 18934 and 35900 unigenes in eggs, larvae, adults and the combined datasets, respectively. Unigenes were annotated using $\mathrm{Nr}$ (NCBI non-redundant protein sequences), Nt (NCBI non-redundant nucleotide sequences), GO (Gene Ontology), PFAM (Protein family), KOG/COG (Clusters of Orthologous Groups of proteins), Swiss-Prot (A manually annotated and reviewed protein sequence database), and KO (KEGG Orthology). Totally 12196 unigenes were annotated into 51 sub-categories belonging to three main GO categories; 8462 unigenes were classified functionally into 26 categories to KOG classifications; 5160 unigenes were functionally classified into 5 KEGG categories. Moreover, according to RSEM, the number of differentially expressed genes between larvae and eggs, adults and eggs, adults and larvae, and the common differentially expressed genes were 2637, 1804, 2628 and 258, respectively. Among them, 17 odorant-binding proteins (OBPs), 7 chemosensory proteins (CSPs) and 8 ionotropic receptors (IRs) were differently expressed in adults and larvae. Ten were confirmed as significant differentially expressed genes. Furthermore, OBP Cmeg32081-c4 was highly expressed in the female head and Cmeg33593_c0 were up-regulated with the increase of larval age.

Conclusions: A comprehensive sequence resource with desirable quality was built by comparative transcriptome of eggs, larvae and adults, enriching the genomic platform of $C$. megacephala. The identified differentially expressed genes would facilitate the understanding of metamorphosis, development and the fitness to environmental change of C. megacephala. OBP Cmeg32081-c4 and Cmeg33593_c0 might play a crucial role in the interactions between olfactory system and biological processes.

Keywords: Chrysomya megacephala, Developmental transcriptome, Olfactory proteins, qPCR

\section{Background}

Chrysomya megacephala is distributed worldwide across all the continents expect Antarctica. It is always found in association with humans or the activities of humans [1-4]. It is a common species found at fresh-food markets, garbage piles, restaurants, school cafeterias, hog and

* Correspondence: zhufen@mail.hzau.edu.cn

Hubei Insect Resources Utilization and Sustainable Pest Management Key Laboratory, Huazhong Agricultural University, Wuhan 430070, China poultry farms and ranches [5]. Excessive populations are not only an irritant to humans but they can also be as disease vectors [6]. By crawling over and feeding on filth, flies become contaminated with pathogens that become entrapped on their legs and body surfaces or taken into the digestive tract with food. In subsequent visits to human food, the flies may leave behind some of these pathogens. Their habit of regurgitating some of their food and expelling feces frequently, both of which 
may contain pathogens, contaminate food, food preparation surfaces and storage containers [7].

In addition to causing annoyance and disease, $C$. megacephala is considered one of the most important species of flies to forensic science. It has long been used as forensic evidences to estimate postmortem interval (PMI) based on the morphological characteristics of individuals collected from the decomposing remains [8]. The ages of its larvae are commonly used as a keystone to achieve accurate PMI of bodies [9]. Therefore, extensive studies have been launched on the population dynamics, oviposition preference and development related studies of C. megacephala [10-15]. In modern facility agriculture $C$. megacephala is an important pollinator for orchards and vegetables, especially for mangos [16]. It is a top pollinator of Diptera which takes up 25\% of all observed Orders in Northern Australia [17]. And in Guangxi province of China, C. megacephala accounts for over $30 \%$ of the total pollinators [18]. Besides, the larvae of C. megacephala are becoming a new sustainable resource for providing animal proteins, lipids, chitosan, and biofuel [19-22]. Despite all this, a key bottle neck to progress in controlling or using C. megacephala is lack of knowledge of the basic molecular biology of this species. Molecular progress of this species will provide important inroads to the discovery of novel target sites for population control, understanding of the immune response in this necrophagous fly. Transcriptome information and the differentially expressed genes related to lipometabolism in response to different kinds of oils were reported in larvae [23]. Despite efforts by developmental biologists, there is little molecular data regarding eggs and adults.

The olfactory system is usually used by insects to locate hosts, oviposition sites, and food sources. Completion of Drosophila genomes and progress in the study of Drosophila olfaction provided unprecedented opportunities to study other Dipterans olfaction. It has been clearly demonstrated that olfactory proteins, including the odorant-binding proteins (OBPs), chemosensory proteins (CSPs), odorant-degrading enzymes (ODEs), odorant receptors (ORs), ionotropic receptors (IRs), and sensory neuron membrane proteins (SNMPs), are involved in the peripheral events of odorant reception [24]. These olfactory proteins are critical for insects to move around and avoid risk factors; and to locate and evaluate food, shelter, mates, and breeding substrates [25]. For C. megacephala, olfactory proteins are vital for it to land premierly and colonize on corpses, and to locate the flowers precisely and feed on nectar, which provides a desirable pollination rate $[17,26,27]$. Identifying functional olfactory molecules will also facilitate development of attractants for baits in management systems.

In the present study, we used RNA-seq to dig the developmental stage-specific genes by building transcriptomes of eggs, larvae from 1st instar to before pupa stage, and adults from emergence to egg laying period (10 days old). We identified differentially expressed genes among eggs, larvae, and adults by comparative transcriptome analysis. We also screened olfactory proteins in this synanthropic fly, including OBPs, CSPs, and IRs, since the olfactory system is usually crucial for insects to locate hosts, oviposition sites, and food sources. Moreover, differential expressed OBPs and CSPs in larvae and adult were testified for transcriptome data validation.

\section{Results}

\section{Illumina sequencing and assembly}

Raw reads with low quality, adapter, and content of $\mathrm{N}$ more than $10 \%$ were excluded to achieve clean reads. Clean reads in eggs, larvae and adults of C. megacephala were 34716158,34347518 , and 35560603, respectively. All clean reads were assembled into transcripts by Trinity software; and the longest copy of redundant transcripts was regarded as a unigene $[28,29]$. Totally, 59486 transcripts were achieved and assembled into 35900 unigenes. Many unigenes had a length between 200-1000 bp (Table 1). Approximately $26.5 \%$ unigenes had a length more than $1000 \mathrm{bp}$ and $12.5 \%$ unigenes had a length more than 2000 bp (Table 1).

\section{Annotation of unigenes}

In order to annotate the unigenes, database $\mathrm{Nr}$ (NCBI nonredundant protein sequences), $\mathrm{Nt}$ (NCBI non-redundant nucleotide sequences), GO (Gene Ontology), PFAM (Protein family), KOG/COG (Clusters of Orthologous Groups of proteins), Swiss-Prot (A manually annotated and reviewed protein sequence database), and KO (KEGG Orthology) were used. Unigenes annotated in CE, CL, CA, CE-specific, CL-specific, CA-specific, Common and CE-CL-CA Combined datasets were 22286, 17180, 18934, 5505, 1711, 2721, 12809 and 35900, respectively (Table 2). For these datasets, number of unigenes annotated in different database and their separate percentage were counted. In the CE-CL-CA Combined dataset, the NR database (13797, 38.43\%) had the largest match (Table 2). The SwissProt (10097, 28.12\%), PFAM (11401, 31.75\%), and GO (12196, 33.97\%) shared similar quantities (Table 2).

Unigenes annotated in eggs, larvae, and adults were shown in supplementary materials with information of

Table 1 Number and length of transcripts and unigenes

\begin{tabular}{lll}
\hline & Transcripts & Unigenes \\
\hline $200-500$ bp & 25787 & 20069 \\
$500-1 \mathrm{k} \mathrm{bp}$ & 10636 & 6300 \\
$1 \mathrm{k}-2 \mathrm{k} \mathrm{bp}$ & 10038 & 5056 \\
$>2 \mathrm{k} \mathrm{bp}$ & 13025 & 4475 \\
Total number & 59486 & 35900 \\
\hline
\end{tabular}


Table 2 Unigenes annotated in different databases

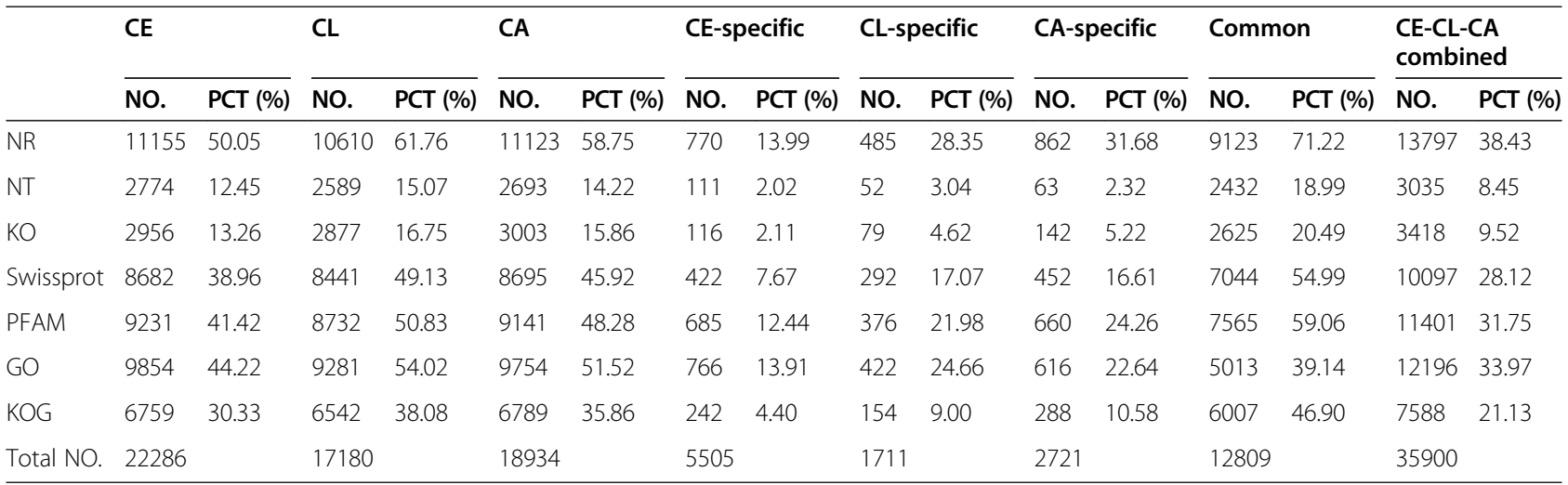

Abbreviations: CE: Unigenes of Chrysomya megacephala eggs; CL: Unigenes of C. megacephala larvae; CA: Unigenes of C. megacephala adults; CE-specific: Specific unigenes of $C$. megacephala eggs; CL-specific: Specific unigenes of $C$. megacephala larvae; CA-specific: Specific unigenes of $C$. megacephala adults; Common: Common unigenes of $C$. megacephala eggs, larvae and adults; $C E-C L-C A$ Combined: Total unigenes of $C$. megacephala eggs, larvae and adults.

NO.: number; PCT (\%): percentage (\%); NR: NCBI non-redundant protein sequences; NT: NCBI non-redundant nucleotide sequences; KO: KEGG Orthology; Swissprot: A manually annotated and reviewed protein sequence database; PFAM: Protein family; GO: Gene Ontology; KOG: Clusters of Orthologous Groups of proteins; Total NO.: Total number of annotated unigenes.

gene's ID, length, reads per kilo bases per million mapped (RPKM) and annotation to different databases (Additional files 1, 2 and 3). The number of the unigenes with RPKM > 0.3 shared by eggs and larvae, larvae and adults, and eggs and adults were 14423, 13823, and 15167, respectively. Eggs, larvae, and adults had 12809 common unigenes (Figure 1).

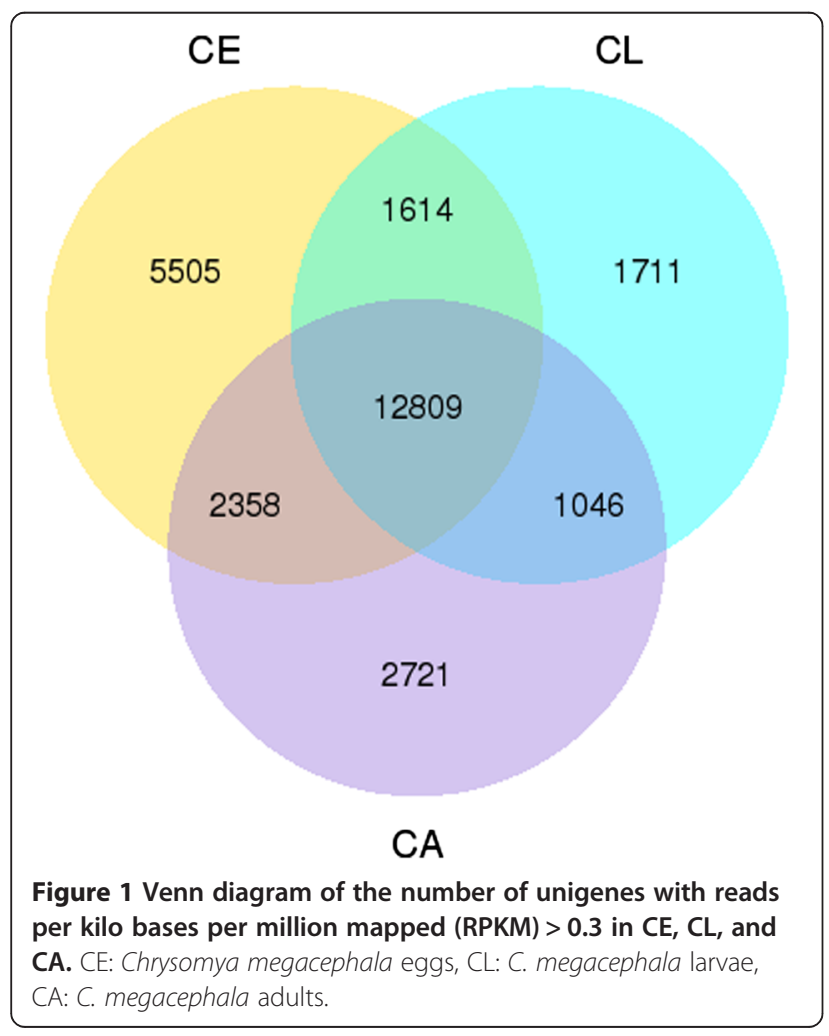

\section{Functional annotation results}

Totally 12196 were annotated into 51 sub-categories belonging to three main GO categories: biological process (BP), cellular component (CC), and molecular function (MF) (Figure 2). There were 23 sub-categories in BP, 17 sub-categories in CC, and 11 sub-categories in MF. Top ten sub-categories were cellular process (7398), metabolic process (6668), single-organism process (4423), biological regulation (2986), cell (4612), cell part (4611), organelle (3235), membrane (2815), binding (7161), and catalytic activity (5343) (Additional file 4).

By KOG classifications, 8462 unigenes were classified functionally into 26 categories (Figure 3). The cluster of 'General Functional Prediction only' was the largest group, which had 1639 unigenes. The group of 'Signal transduction' was in second place, which had 1115 unigenes. Top 2 categories had $32.5 \%$ of unigenes annotated to KOG database (Additional file 5).

In total, 5160 unigenes were functionally classified into 5 KEGG categories (Figure 4). They were cellular processes (746 unigenes, $13.95 \%$ of the unigenes annotated to the KEGG database), environmental information processing $(649,12.14 \%)$, genetic information processing (943, 17.63\%), metabolism (1760, 32.91\%), and organismal systems (1250, 23.37\%) (Additional file 6). Among 31 subcategory, 'signal transduction', 'translation', 'transport and catabolism' were the top 3.

\section{Differentially expressed genes}

Differentially expressed genes were selected by RSEM with conditions of $\log _{2}$ Fold change $>1$ and $q$ value $<0.005$ [30] The number of differentially expressed genes between larvae and eggs, adults and eggs, and adults and larvae 


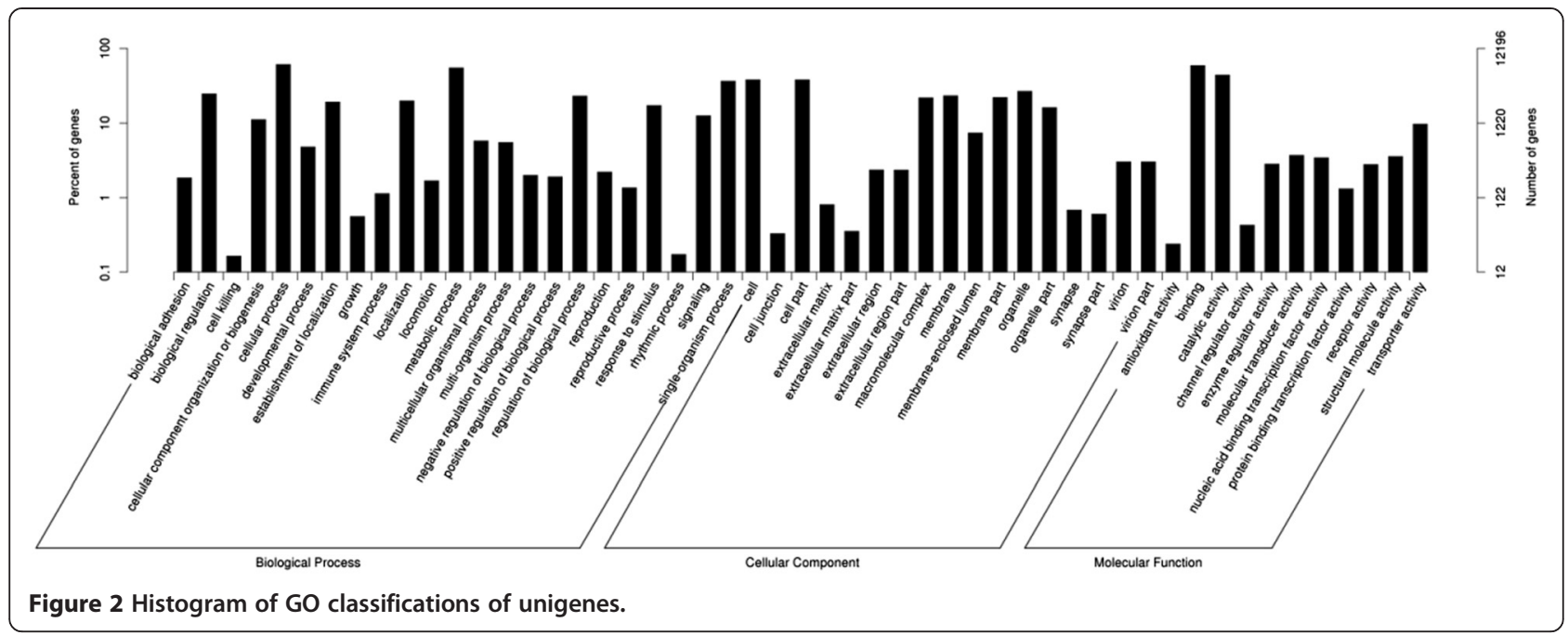

were 2637 , and 1804, 2628, respectively (Additional files 7 , 8 and 9). There were 258 common differentially expressed genes among eggs, larvae, and adults (Figure 5). Totally, 1280 differentially expressed genes in larvae and eggs were also differential expressed in adults and eggs. And 864 differentially expressed genes in larvae and eggs were also differential expressed in adults and larvae (Figure 5). We also found that 974 differentially expressed genes in adult and egg were differential expressed in adults and larvae (Figure 5). More expressed genes in larvae than in eggs, in adults than in eggs, and in adults than in larvae were 1255, 1150, and 836, respectively (Figure 6). But less expressed genes in larvae than in eggs, in adults than in eggs, and in adults than in larvae were 1013, 1847, and 968, respectively (Figure 6).

\section{Expression profiles of olfactory proteins}

We identified 49 OBPs, 12 CSPs, and 11 IRs through $\mathrm{Nr}$ database (Nucleotide sequences were listed in the Additional files 10, 11 and 12). Seventeen OBPs, 7 CSPs, and 8 IRs were differently expressed in adults and larvae (Table 3). Only 9 OBPs and 1 CSP were significantly differently expressed (Table 3 ).

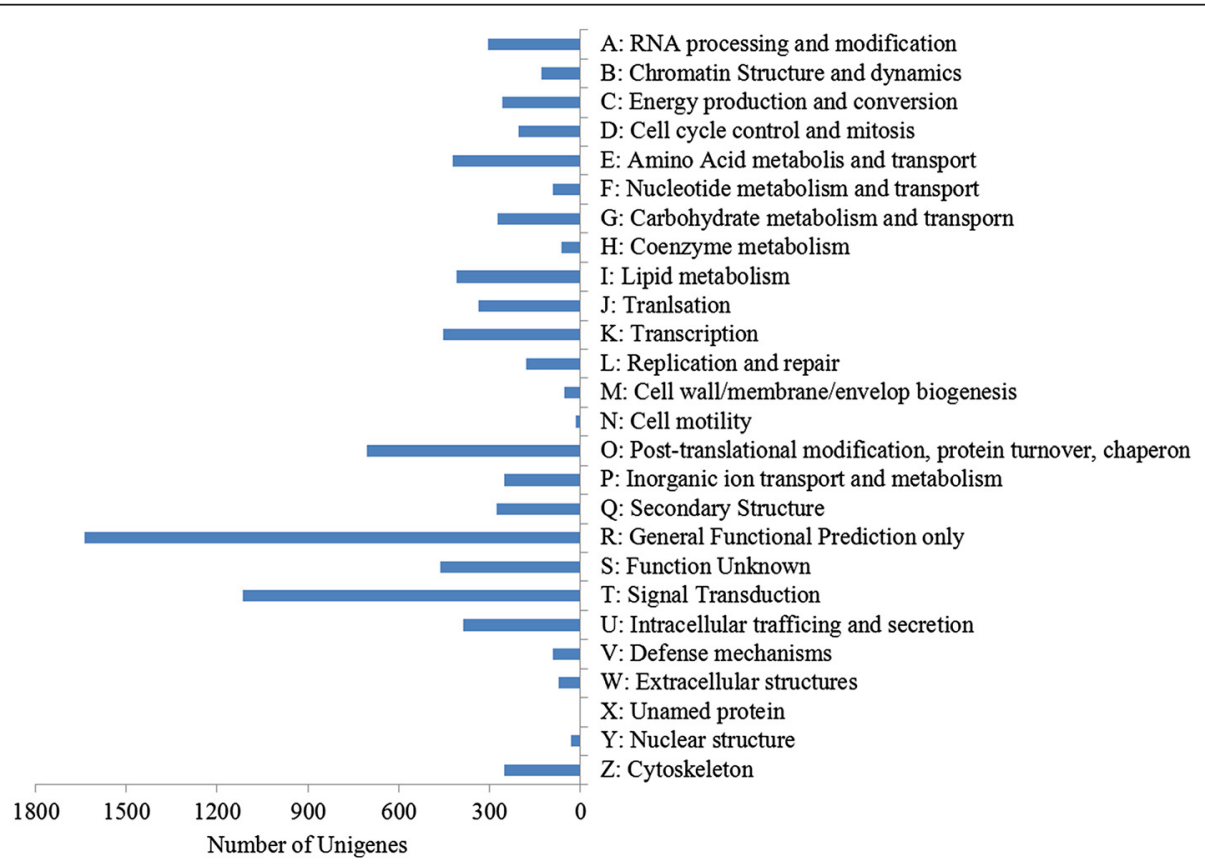

Figure 3 Histogram of KOG classifications of unigenes. 


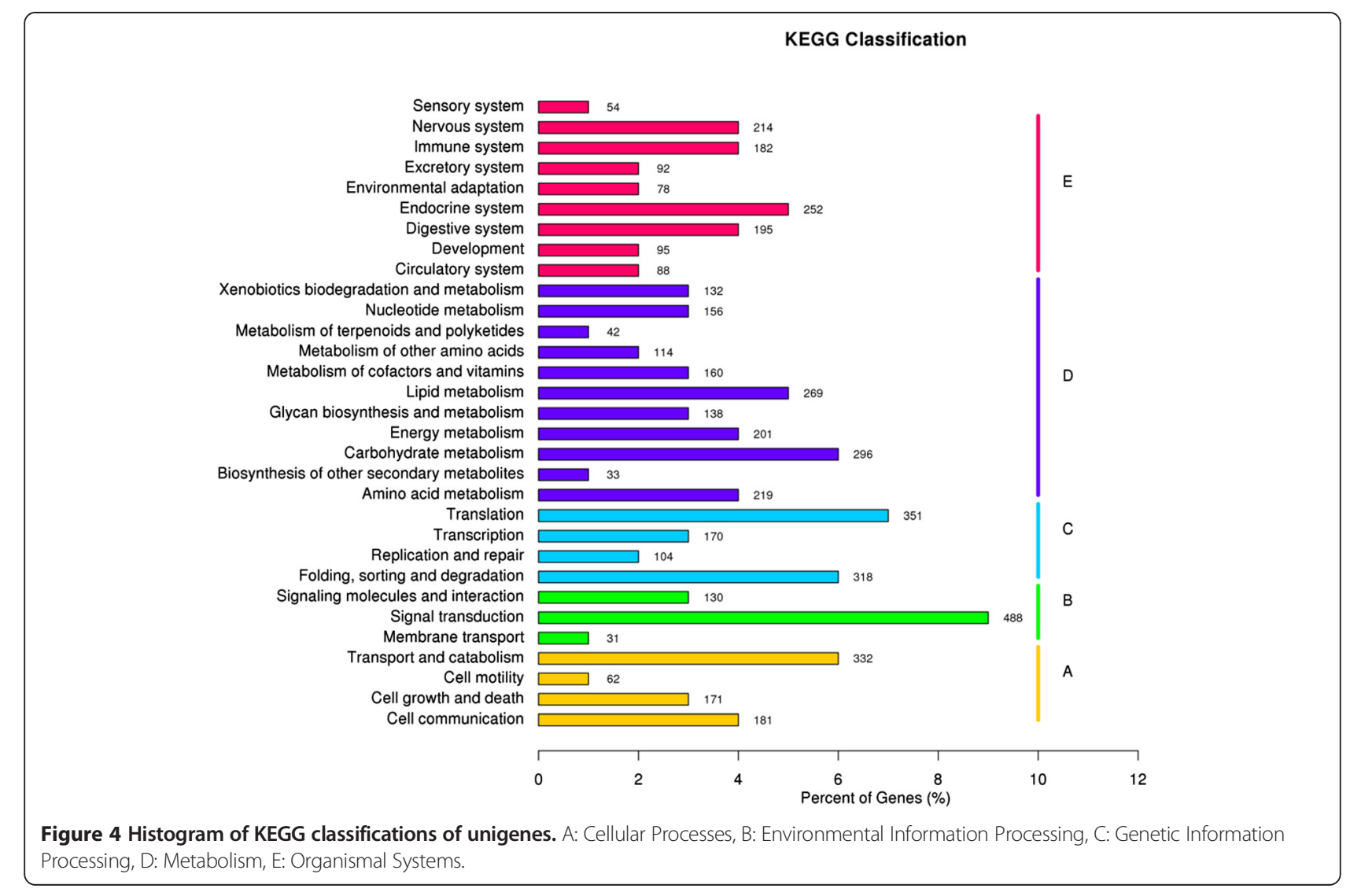

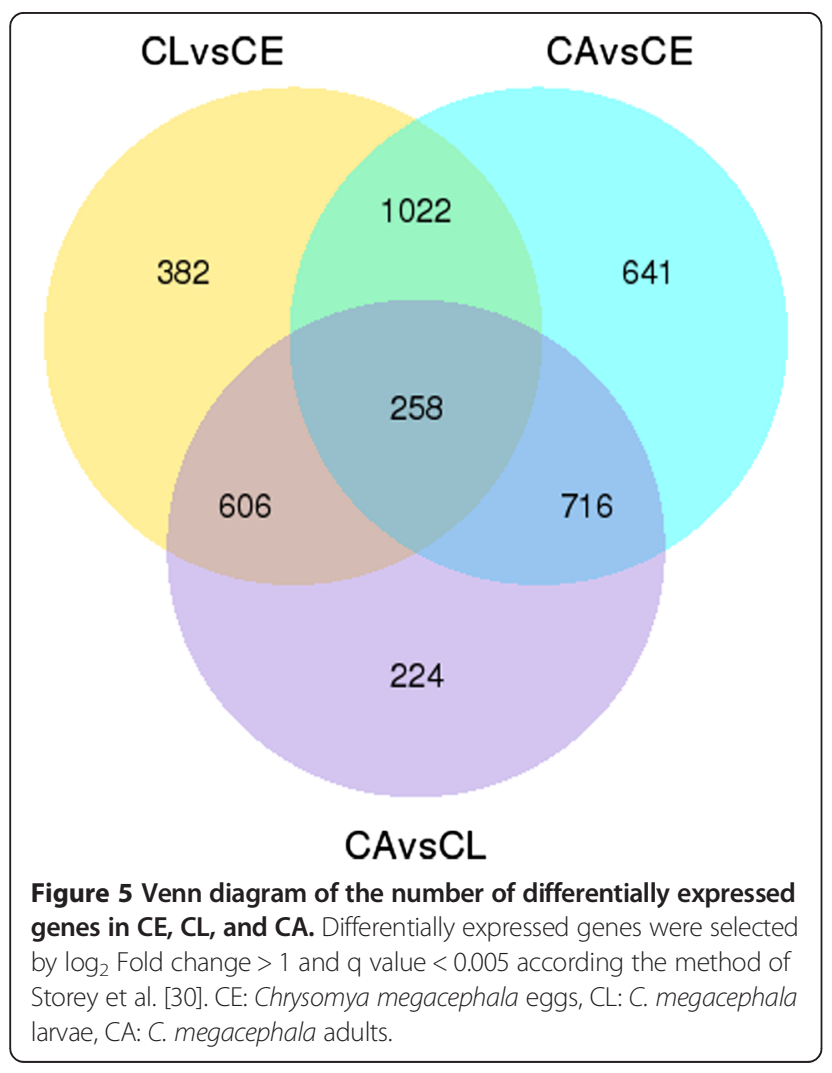

\section{Validation of transcriptome data by $\mathrm{qPCR}$}

To validate the transcriptome result, we selected 10 significant differentially expressed genes from Table 2 for quantitative real-time PCR (qPCR) conformation. The primers used for qPCR were shown in Additional file 13. The result of qPCR was shown in Figure 7. Seven OBPs transcripts and one CSP transcript which have demonstrated by RNA-seq to be enriched in larvae were confirmed by qPCR. The expression proportion of the 8 genes in adult to larva varied from the least 1.6\% (OBP Cmeg31019_c2, Figure 7F) to the biggest 40.15\% (CSP Cmeg21206_c0, Figure 7J). Additionally, RNA-seq data for two OBPs, Cmeg23484-c0 and Cmeg32081-c4, enriched in adults mirrored the qPCR data (Figure 7). Cmeg23484- $\mathrm{cO}$ and Cmeg32081-c4 had significantly higher transcriptional level in adult than in larva with 2.64 and 113.67 fold exchanges, respectively. Moreover, the tissue-specific expression pattern of Cmeg32081-c4 in female and the larval developmental expression pattern of Cmeg33593-c0 were performed (Figure 8). OBP Cmeg32081-c4 was highly expressed in the head of the female (Figure $8 \mathrm{~B}$ ) and Cmeg33593_c0 were up-regulated with the increase of larval age (Figure 8A). 

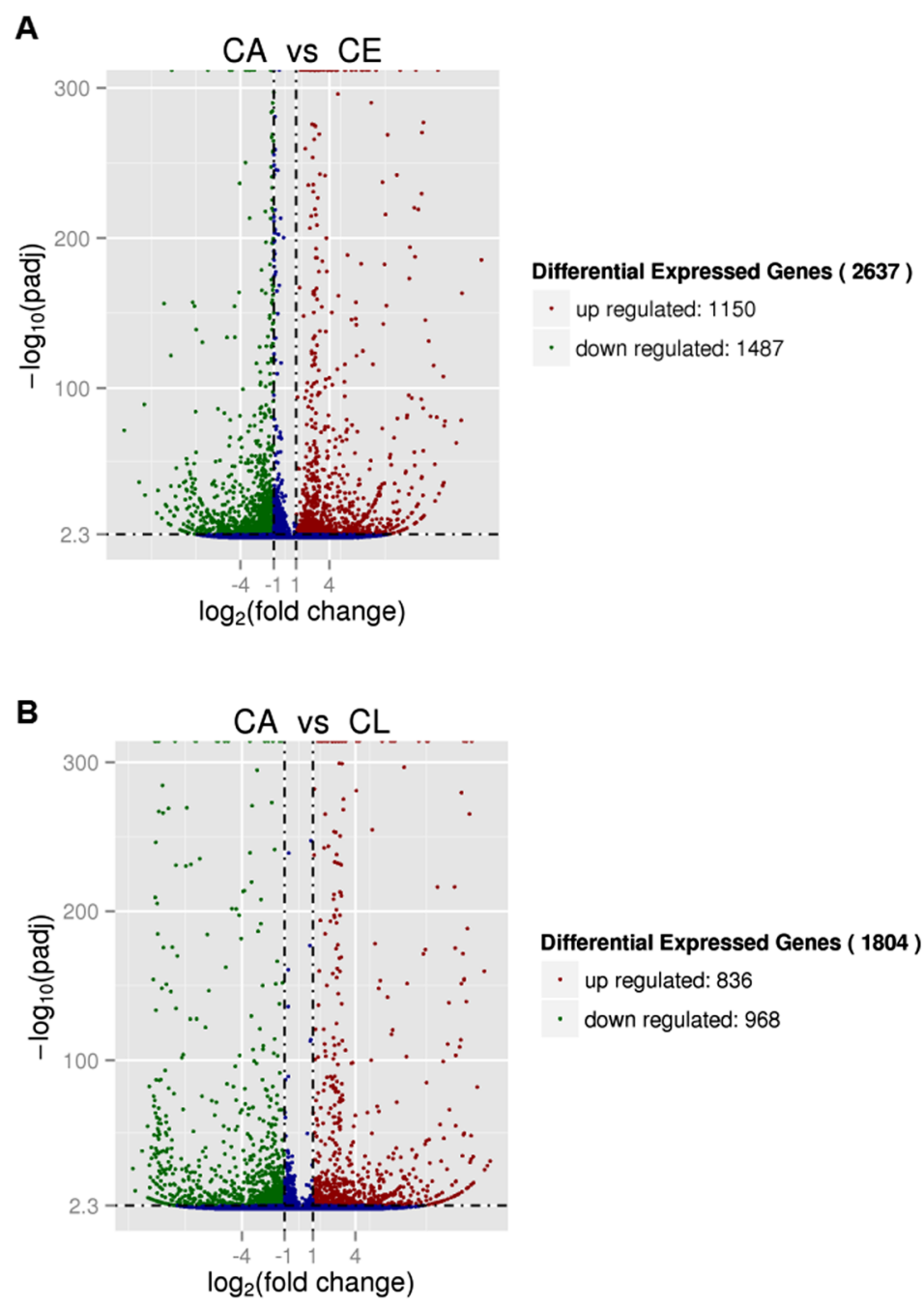

Differential Expressed Genes ( 1804 )

- up regulated: 836

- down regulated: 968

C

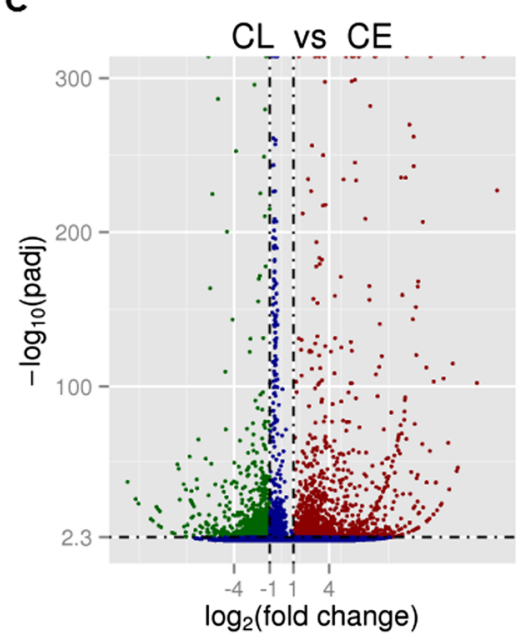

Differential Expressed Genes ( 2268 )

- up regulated: 1255

- down regulated: 1013

Figure 6 (See legend on next page.) 
(See figure on previous page.)

Figure 6 Volcano plot of differentially expressed genes in eggs, larvae, and adults. A: Volcano plot of differentially expressed genes between CA and CE. B: Volcano plot of differentially expressed genes between CA and CL. C: Volcano plot of differentially expressed genes between CL and CE. Differentially expressed genes were selected by log2 Fold change $>1$ and q value $<0.005$ according the method of Storey et al. [30]. Splashes represent different genes. Blue splashes means genes without significant different expression. Red splashes means significantly up expressed genes. Green splashes means significantly down expressed genes. CE, CL, and CA represent eggs, larvae and adults of Chrysomya megacephala, respectively.

Table 3 Differential expressed olfactory-related genes in adults and larvae

\begin{tabular}{|c|c|c|c|c|c|}
\hline & Gene & Readcount_Adult & Readcount_Larva & $\log _{2}$ Fold_change & $q$ \\
\hline \multirow[t]{17}{*}{$\overline{\mathrm{OBPS}}$} & Cmeg21243_c0 & 2.286 & 1.4019 & 0.7054 & $>0.005$ \\
\hline & Cmeg21269_c0 & 32.7183 & 854.4521 & -4.7068 & $<0.005^{*}$ \\
\hline & Cmeg21549_c0 & 5.2439 & 0.163 & 5.0076 & $>0.005$ \\
\hline & Cmeg21654_c0 & 0.9608 & 2.3474 & -1.2888 & $>0.005$ \\
\hline & Cmeg23484_c0 & 219.3585 & 95.1018 & 1.2057 & $<0.005^{*}$ \\
\hline & Cmeg24919_c0 & 71.1444 & 49.996 & 0.5089 & $>0.005$ \\
\hline & Cmeg25217_c1 & 1.1264 & 0.0326 & 5.1106 & $>0.005$ \\
\hline & Cmeg27557_c0 & 4.0088 & 4.0754 & -0.0238 & $>0.005$ \\
\hline & Cmeg28108_c1 & 0.762 & 12.9442 & -4.0864 & $<0.005^{*}$ \\
\hline & Cmeg28677_c0 & 14.3274 & 2.6083 & 2.4576 & $>0.005$ \\
\hline & Cmeg29057_c0 & 11.9602 & 107.3274 & -3.1657 & $<0.005^{*}$ \\
\hline & Cmeg30479_c0 & 5.8973 & 231.3797 & -5.2941 & $<0.005^{*}$ \\
\hline & Cmeg31019_c2 & 9.163 & 94.0392 & -3.3594 & $<0.005^{*}$ \\
\hline & Cmeg32081_c4 & 20.6073 & 0.4891 & 5.397 & $<0.005^{*}$ \\
\hline & Cmeg33593_c0 & 788.7731 & 2558.267 & -1.6975 & $<0.005^{*}$ \\
\hline & Cmeg8311_c0 & 303.3185 & 477.3415 & -0.6542 & $>0.005$ \\
\hline & Cmeg8717_c0 & 2.1535 & 50.6347 & -4.5554 & $<0.005^{*}$ \\
\hline \multirow[t]{10}{*}{ CSPs } & Cmeg21206_c0 & 1559.024 & 361.927 & 2.1069 & $<0.005^{*}$ \\
\hline & Cmeg25565_c0 & 2.1402 & 0.0529 & 5.3383 & $>0.005$ \\
\hline & Cmeg23554_c0 & 29.416 & 33.3811 & -0.1824 & $>0.005$ \\
\hline & Cmeg349412_c0 & 0.8719 & 0.0265 & 5.0428 & $>0.005$ \\
\hline & Cmeg30884_c0 & 3.8048 & 4.0769 & -0.0997 & $>0.005$ \\
\hline & Cmeg5343_c0 & 0.0793 & 0.1058 & -0.4166 & $>0.005$ \\
\hline & Cmeg645582_c0 & 0.1585 & 0.0265 & 2.5834 & $>0.005$ \\
\hline & Cmeg386817_c0 & 0.1982 & 0.3174 & -0.6797 & $>0.005$ \\
\hline & Cmeg425837_c0 & 0.1982 & 0.2116 & -0.0947 & $>0.005$ \\
\hline & Cmeg23554_c0 & 29.416 & 33.3811 & -0.1824 & $>0.005$ \\
\hline \multirow[t]{8}{*}{ IRs } & Cmeg1881_c0 & 0.1585 & 0.5026 & -1.6645 & $>0.005$ \\
\hline & Cmeg20304_c0 & 0.1982 & 0.0529 & 1.9053 & $>0.005$ \\
\hline & Cmeg22717_c0 & 0.1189 & 0.0265 & 2.1684 & $>0.005$ \\
\hline & Cmeg25409_c2 & 0.1585 & 0.3703 & -1.224 & $>0.005$ \\
\hline & Cmeg25409_c3 & 0.1982 & 0.3968 & -1.0016 & $>0.005$ \\
\hline & Cmeg18139_c0 & 0.0793 & 0.2381 & -1.5865 & $>0.005$ \\
\hline & Cmeg3349_c1 & 0.0793 & 0.1587 & -1.0016 & $>0.005$ \\
\hline & Cmeg475644_c0 & 0.4756 & 0.3174 & 0.5834 & $>0.005$ \\
\hline
\end{tabular}

Note: $q$ value was calculated according the method of Storey et al., $2003 .{ }^{*} \mathrm{q}<0.005$ meant significantly different. 

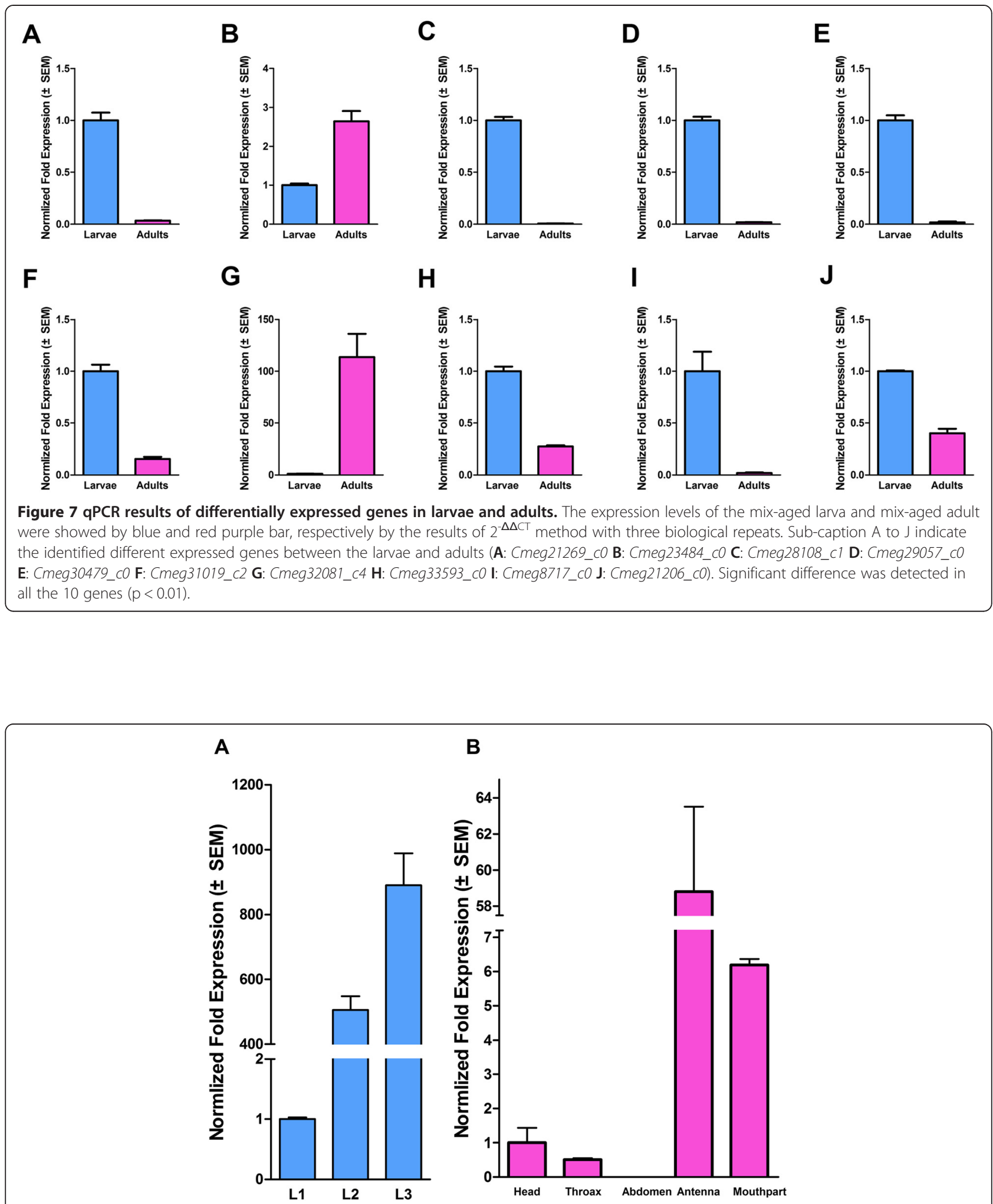

B

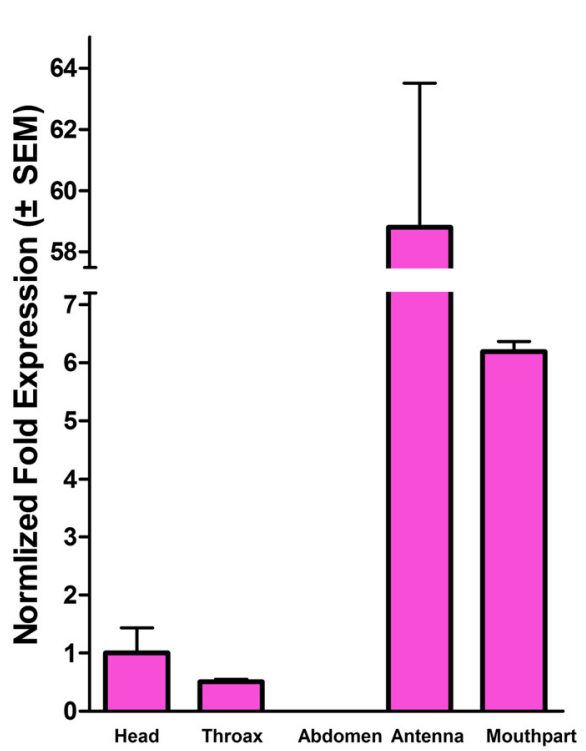

Figure 8 Developmental expression patterns of Cmeg33593_c0 in C. megacephala larvae and tissue-specific expression patterns of Cmeg32081_c4 in C. megacephala females. The expression levels of Cmeg33593_c0 in different instar of larvae were showed red purple bar by the results of $2^{-\Delta \Delta C T}$ method with three biological repeats $(\mathbf{A})$. The expression levels of Cmeg32081_c4 in various tissues of female were showed red purple bar by the results of $2^{-\Delta \Delta C T}$ method with three biological repeats (B). And the column title L1, L2 and L3 represent 1st, 2nd and 3rd instar of larvae, respectively. 


\section{Discussion}

\section{Overview of transcriptome data}

Developmental transcriptomes were established of eggs, mix-aged larvae, and mix-aged adults, providing a relatively comprehensive gene pool of C. megacephala. The number of clean reads in larva transcriptome was 34347518, which was similar to that of a reported larval transcriptome of C. megacephala [31]. And the number of clean reads from egg and adult transcriptome were 34,716,158, and $35,560,603$, respectively. All these clean reads were assembled into 59486 transcripts by Trinity software. Transcripts were assembled into 22286, 17180, 18934 and 35900 unigenes in eggs, larvae, adults and the combined datasets, respectively. A total of 35900 unigenes were annotated by Nr, Nt, GO, PFAM, KOG/COG, Swiss-Prot, and KO. Moreover, thousands of different expressed and common genes between larvae and eggs, adults and eggs, adults and larvae and all three stages were harvested, which both facilitate future developmental and evolutionary studies of $C$. megacephala, and contribute to future work in blowfly comparative genomic. Ten of the identified differentially expressed genes were validated by qPCR, showing that the quality of the transcriptome was desirable.

\section{Olfactory proteins}

Striking similarities span a phylogenetically broad array in olfaction of insects, implying that there is an optimal solution to the problem of detecting and discriminating odors [32]. Therefore, the research into the parallel OBPs in Drosophila melanogaster would provide valuable information to the link the biological roles into the candidate OBPs [25]. Moreover, olfactory proteins have been illustrated to act in the insect nutrient uptake, life span and behavior change during developmental stages $[33,34]$. The developmental transcriptome of $C$. megacephala would be an opportunity to understand the interactions between olfactory proteins anddevelopment. Totally 49 OBPs, 12 CSPs, and 11 IRs were identified. Moreover, a phylogenetic wheel was made based on (deduced) amino acids from D. melanogaster OBPs and C. megacephala OBPs (Additional files 14 and 15), since the identified OBPs had the largest number. We also identified some ODEs, ORs and SNMPs, however they were relatively incomplete, therefore we did not take them out for further analysis. This might be rooted into the sampling characteristics and the abundance of the related genes. Seventeen OBPs, 7 CSPs, and 8 IRs were differently expressed in adults and larvae, since the olfactory systems function mostly in adults and larvae.

Among the 10 significant different olfactory genes, two OBPs genes were more abundant in adults than in larvae. One is Cmeg23484-cO, and the other is Cmeg32081-c4. Cmeg23484-c0 showed $91 \%$ identities with Calliphora stygia OBP (AID61300), 91\% identities with Delia antiqua OBP (BAN59723), and 64\% identities with D. melanogaster OBP44a DmelOBP19d (Alignment results were showed in the Additional file 16). BAN59723 were functionally annotated as an insect pheromone/odor binding protein domains. And DmelOBP44a of adult decreased in expression with increasing organism age, which was considered to be a link between the olfactory sensation and aging [35]. DmelOBP44a was detected in the female antenna extract but not male, which might reflect true sexual dimorphism in the expression of OBPs [36]. The other one Cmeg32081-c4, which showed highest fold changes, was highly expressed in female head, especially in antenna and mouthpart (Figure 8B). Cmeg32081-c4 showed 71\% identities with C. stygia OBP (AID61308) and 38\% identities with of DmelOBP19d (ACY93747) (Alignment results were showed in the Additional file 16). Both DmelOBP44a and DmelOBP19d are the most abundant OBPs in adult antenna extracts by LC/MS/MS $[36,37]$. DmelOBP19d was also expressed in the head at different levels and was considered to have a close connection to the variation in life span associated with nutrient sensing and synaptic transmission by network analysis [33,38]. The function of DmelOBP44a and DmelOBP19d should have an instructive role for the research of Cmeg23484-c0 and Cmeg32081-c4 in aging and nutrient sensing.

Seven OBPs and 1 CSP were found more abundant in larvae than in adults. Among them, OBP Cmeg33593_co has the highest RPKM value, indicating that it has the highest expression level in larvae [39]. Cmeg33593_c0 showed 88\% identities with OBP C. stygia AID61305 and 56\% identities with DmelOBP99b (ABW78474) (Alignment results were showed in the Additional file 16). However, the expression of DmelOBP99b has been well documented in various developmental and physical situations in adults. DmelOBP99b was found to be more abundant in females than males [40]. And DmelOBP99b showed strong adultbiased expression and altered expression levels during aging in both sexes, but in opposite directions: the expression level of young virgin Drosophila females was lower than that of the old ones, while the expression level of the young virgin males was higher than that of the old ones [41]. For males, DmelOBP99b was up-regulated after courting females and down and regulated after mating [41,42]. Moreover, DmelOBP99b was down-regulated after being starved [43]. Therefore, DmelOBP99b was suggested to be sensitive to and probably influence nutrient status and reproductive status in both males and females [44]. For example, the ectopic expression of DmelOBP99b could reduce virgin female receptivity and copulation frequency [45]. Moreover, DmelOBP99b in adults has a wide and comprehensive influence in aversive tastants uptake, which should have evolved to prevent ingestion of toxic compounds [46]. In the UAS-OBP99b-RNAi adults, the 
consumption of berberine and papaverine compared to the control were raised higher by $47 \%$ respectively, and the coumarin and denatonium were decresed by $23 \%$ and $40 \%$ respectively [46]. In addition, the combinatorial response profiles in females and males were diverse for intake of bitter tastants with this line [46]. The discriminative binding profiles between sexes should have a natural tie with the varied expression patterns of DmelOBP99b, which calls for more experiments, guiding the behavior of males and females especially during the copulation.

There should be a certain relationship between OBPs and ORs in odorant detection by comparing behavioral response profiles of OBPs and molecular response profiles of odorant receptors and features of functional organization emerge between behavioral response profiles of OBPs and electrophysiological response profiles of odorant receptors $[37,47,48]$. And the binding function of DmelOBP99b has been well studied. In male it is responsible for the binding of E2-hexenal, acetophenone, benzaldehyde, citral and d-carvone, while in female it is responsible for 2-ethylpyrazine, acetophenone, benzaldehyde, citral and d-carvone [37]. For example, OR10a and OR67a are activated by acetophenone and benzaldehyde, which were identified as bioactive compounds of most floral volatiles [49]. And the behavior response is affected by the suppression of DmelOBP99b for both females and males. And benzaldehyde and E2-hexenal were identified from the volatile organic compounds (VOCs) [50,51]. And acetophenone and benzaldehyde both have a similar structure feature of benzoyl chemical groups [37]. So probe into Cmeg33593_c0 might help to understand the localization of hosts, oviposition sites and food sources, mating behavior and the connection between OBPs and life span.

Though DmelOBP99b in adult was well studied, the expression and function of larvae were scarce. While, it is interesting that the expression of DmelOBP99b-like OBP Cmeg33593_c0 increased with larval growth, which was found occasionally (Figure 8A). It seems that Cmeg33593_c0 was accumulated during larval stages and consumed in adults. According to our observations, the odors from the feeding container increased during the sampling days of larvae. It could be easily explicable because the feedstuff (fish meat) decayed gradually and deeply. Then how to understand the connections between the denser odor and the increasing Cmeg33593_c0? Would Cmeg33593_c0 be a protective amino acid to eliminate the affect of the offensive VOCs, since the parallel of Cmeg33593_c0, DmelOBP99b has a broad odor-binding profile? And anther explanation: together with all information of DmelOBP99b in adult, the increase of Cmeg33593_c0 during larval stages might be bound up with aging, the nutrient accumulation and feeding behavior, which might play a role in adult physiological status. More experiments are needed to illustrate the crosstalk between the olfactory proteins and the developmental genes. The probe into Cmeg33593_co is to throw out a minnow to catch a whale. More significant work could be launched by the established sequence platform, which would facilitate the illustration of the crosstalk between the olfactory proteins and development, the application of pollination and forensic science in $C$. megacephala, avoid potential transmission of pathogens.

\section{Conclusions}

A comprehensive sequence resource with desirable quality was built by developmental transcriptomes of eggs, larvae and adults, enriching the genomic platform, which would facilitate the understanding of metamorphosis, development and the fitness to environmental change of $C$. megacephala. The identified OBP Cmeg32081-c4 and Cmeg33593_c0 might play a crucial role in the interactions between olfactory system and physiological status.

\section{Methods \\ RNA sequencing}

Eggs, mixed larvae from 1st instar to before pupa stage, and mixed adults from emergence to egg laying period (10 days old) were prepared for RNA extraction. RNA purity was checked using the NanoPhotometer ${ }^{\circ}$ spectrophotometer (IMPLEN, CA, USA). And RNA integrity was assessed using the RNA Nano6000 Assay Kit of the Agilent Bioanalyzer 2100 system (Agilent Technologies, CA, USA). A total amount of $3 \mu \mathrm{g}$ RNA per sample was used as input material for the RNA sample preparations. Briefly, mRNA was purified from total RNA using poly-T oligo-attached magnetic beads. Sequencing libraries were generated using NEBNext ${ }^{\circ}$ Ultra $^{\mathrm{Tm}}$ RNA Library Prep Kit for Illumina ${ }^{\circ}$ (NEB, USA) following manufacturer's recommendations and index codes were added to attribute sequences to each sample. Library quality was assessed on the Agilent Bioanalyzer 2100 system. The clustering of the index-coded samples was performed on a cBot Cluster Generation System using TruSeq PE Cluster Kit v3-cBotHS (Illumia) according to the manufacturer's instructions. After cluster generation, the library preparations were sequenced on an Illumina Hiseq2000 platform and 100 paired-end reads were generated.

\section{Transcriptome data analysis}

Raw data (raw reads) of fastq format were firstly processed through in-house perl scripts. Clean reads were obtained after removing reads that contained adaptor sequences, reads in which more than $10 \%$ of the bases were unknown, and reads in which more than $50 \%$ of the quality values of the bases were less than 5 . At the same time, Q20, Q30, GC-content and sequence duplication level of 
the clean data were calculated. All the downstream analyses were based on clean data with high quality. The left files (read1 files) from all libraries/samples were pooled into one big left.fq file, and right files (read2 files) into one big right.fq file. Transcriptome assembly was accomplished based on the left.fq and right.fq using Trinity with min_kmer_cov set to 2 by default and all other parameters set default $[28,29]$. Unigenes were used for BLAST searches with annotation against the $\mathrm{Nr}$ database using an E-value cut-off of $10^{-5}$ (E-value $<0.00001$ ). After sequence assembly, the unigene sequences were also aligned by BLASTX to protein databases such as SwissProt, KEGG and COG, in order to retrieve proteins with the highest sequence similarity to the given unigenes along with putative functional annotations. Gene function was annotated based on the following databases: $\mathrm{Nr}, \mathrm{Nt}$, Pfam, KOG/COG, Swiss-Prot, KO and GO.

\section{qPCR}

Total RNA was extracted from 1st, 2nd and 3rd instar larvae for larval stage expression. Adult females on ice were quickly dissected into head (without antenna and mouthpart), thorax, abdomen, antenna and mouthpart. Tissues were immediately transferred into liquid nitrogen before RNA extraction. Total RNA from each sample was extracted using TRIzol $^{\circ}$ Reagent (Ambion', Life technologies, U.S.) according to the manufacturer's protocol. Concentration and quality of each RNA sample was determined by Nanodrop2000 (Thermo Scientific, U.S.). Samples were allowed into further experiments with an appropriate OD260/280 value from 1.9 to 2.1. One $\mu \mathrm{g}$ of total RNA from each sample was applied to produce the first strand cDNA with First Strand cDNA Synthesis Kit (NEWBIO Tech., Canada) according to the manufacturer's protocol. All cDNA was stored at $-20^{\circ} \mathrm{C}$ before use.

The primers were designed with IDT online tools (http:// www.idtdna.com/scitools/Applications/RealTimePCR/) and listed in the Additional file 13. RT-PCR was applied to test the primers' quality of differentially expressed genes. Primer pairs led to the PCR products exact identities between the RT-PCR fragments and transcripts from RNA-seq were used for qPCR. RT-PCR was performed as follows: $95^{\circ} \mathrm{C}$ for $3 \mathrm{~min}, 35$ cycles at $95^{\circ} \mathrm{C}$ for $30 \mathrm{sec}$, $57^{\circ} \mathrm{C}$ for $30 \mathrm{sec}$ and $72^{\circ} \mathrm{C}$ for $20 \mathrm{sec}$; and final extension at $72^{\circ} \mathrm{C}$ for $5 \mathrm{~min}$. qPCR was performed using Real Master Mix (SYBR Green) (NEWBIO Tech., Canada) on a BioRad iQ5 Optical System (Bio-Rad). The procedure for qPCR were as follows: initial denaturation temperature, $95^{\circ} \mathrm{C}$ for $30 \mathrm{sec}$, followed by 40 cycles at $95^{\circ} \mathrm{C}$ for $5 \mathrm{sec}$ and $59^{\circ} \mathrm{C}$ for $30 \mathrm{sec}$, and $72^{\circ} \mathrm{C}$ for $2 \mathrm{~min}$ to terminate the reaction. After the reaction, a melting curve analysis from $55^{\circ} \mathrm{C}$ to $95^{\circ} \mathrm{C}$ was applied to ensure consistency and specificity of the amplified product. Gene $\alpha$-tubulin (GenBank: KM289152) was used as positive control to test the quality of cDNA. Elongation factor 1 (EMBL: FR719225) and RPL8 (GenBank: KM289151) of C. megacephala were used as reference genes in qPCR of Cmeg32081-c4 and Cmeg33593_c0, respectively (according to our previous work, unpublished). For each treatment, three biological replicates were conducted. Data of qPCR was analyzed by $2^{-\Delta \Delta \mathrm{CT}}$ method.

\section{Additional files}

Additional file 1: S1-annotation Eggs.
Additional file 2: S2-annotation Larvae.
Additional file 3: S3-annotation Adults.
Additional file 4: S4-SC1 GO.
Additional file 5: S5-SC2 KOG.
Additional file 6: S6-SC3 KEGG.
Additional file 7: S7-SD1 CAvsCE.
Additional file 8: S8-SD2 CAvsCL.
Additional file 9: S9-SD3 CLvsCE.
Additional file 10: S11-nucleotide sequences of OBPs.
Additional file 11: S12-nucleotide sequences of CSPs.
Additional file 12: S13- nucleotide sequences of IRs.
Additional file 13: S10-Primers of qPCR for C. megacephala used in
the experiment.
Additional file 14: S14-deduced amino acid sequences of the
identified OBPs.
Additional file 15: S15-Phylogenetic analysis of OBPs of Chrysomya
megacephala and Drosophila melanogaster.
Additional file 16: S16-Mutiple alignment of deduced amino acid of
the Cmeg23484_c0, Cmeg32081_c4 and Cmeg33593_c0.

\section{Competing interests}

The authors declare that they have no competing interests.

\section{Authors' contributions}

This experiment was conceived and coordinated by XW, FZ, CL and XM in this study. Sampling was performed by XW and MX. Transcriptome data analysis was performed by XW and FZ. Sequence analysis was performed by XW and MX. RT-PCR and qPCR were performed by XW and MX. XW and FZ drafted the manuscript. All authors read and approved the final version of the manuscript.

\section{Supporting information}

The blowfly Chrysomya megacephala transcriptome data were submitted to the Sequence Read Archive (SRA) of the NCBI (accession number: SRP050024).

\section{Acknowledgements}

We acknowledge the Novogene at Beijing for its assistance in original data processing and related bioinformatics analysis. We also thank Rong Yuan for her help with the insect rearing and sampling; and Colin Wong for his valuable suggestions with the manuscript revision. This work was partly supported by EU-funded project PROtelNSECT (Grant No.034082) and the Fundamental Research Funds for the Central Universities (Grant No.2014PY059).

Received: 1 October 2014 Accepted: 22 December 2014 Published online: 23 January 2015

\section{References}

1. Tomberlin JK, Reeves WK, Sheppard DC. First record of Chrysomya megacephala (Diptera: Calliphoridae) in Georgia, USA. Fla Entomol. 2001;84(2):300.

2. Martinez-Sanchez A, Marcos-Garcia M, Rojo S. First collection of Chrysomya megacephala (Fabr.) in Europe (Diptera: Calliphoridae). Pan-Pac Entomol. 2001;77(4):240-3. 
3. Wells JD. Chrysomya megacephala (Diptera: Calliphoridae) has reached the continental United States: review of its biology, pest status, and spread around the world. J Med Entomol. 1991;28(3):471-3.

4. Castro e CP, García M. First record of Chrysomya megacephala (Fabricius, 1794) (Diptera, Calliphoridae) from Portugal. World. 2009;25:199-200.

5. Chaiwong T, Srivoramas T, Sueabsamran P, Sukontason K, Sanford MR Sukontason KL. The blow fly, Chrysomya megacephala, and the house fly, Musca domestica, as mechanical vectors of pathogenic bacteria in Northeast Thailand. Trop Biomed. 2014;31(2):336-46.

6. Ferraz ACP, Proenca B, Gadelha BQ, Faria LM, Barbalho MGM, Aguiar-Coelho VM, et al. First Record of Human Myiasis Caused by Association of the Species Chrysomya megacephala (Diptera: Calliphoridae), Sarcophaga (Liopygia) ruficornis (Diptera: Sarcophagidae), and Musca domestica (Diptera: Muscidae). J Med Entomol. 2010:47(3):487-90

7. Srivoramas T, Chaiwong T, Sanford MR. Isolation of fungi from adult house fly; Musca domestica and the blow fly Chrysomya megacephala in ubon ratchathani province, Northeastern Thailand. Int J Parasitol Res. 2012;4(1):4

8. Erzinclioglu Y. Forensic entomology and criminal investigations. Police J. 1991;64:5.

9. Catts EP, Goff ML. Forensic entomology in criminal investigations. Annu Rev Entomol. 1992:37(1):253-72.

10. Yang S-T, Shiao S-F. Oviposition preferences of two forensically important blow fly species, Chrysomya megacephala and C. rufifacies (Diptera: Calliphoridae), and implications for postmortem interval estimation. J Med Entomol. 2012;49(2):424-35.

11. Thevan K, Ahmad AH, Rawi CSM, Singh B. Growth of Chrysomya megacephala (Fabricius) Maggots in a Morgue Cooler. J Forensic Sci. 2010;55(6):1656-8

12. Rezende F, Alonso MA, Souza CM, Thyssen PJ, Linhares AX. Developmental rates of immatures of three Chrysomya species (Diptera: Calliphoridae) under the effect of methylphenidate hydrochloride, phenobarbital, and methylphenidate hydrochloride associated with phenobarbital. Parasitol Res. 2014;113(5):1-11.

13. Souza CM, Thyssen PJ, Linhares AX. Effect of nandrolone decanoate on the development of three species of Chrysomya (Diptera: Calliphoridae), flies of forensic importance in Brazil. J Med Entomol. 2011:48(1):111-7.

14. Oliveira HG, Gomes G, Morlin Jr JJ, Von Zuben CJ, Linhares AX. The effect of Buscopan ${ }^{\oplus}$ on the Development of the Blow Fly Chrysomya megacephala (F.) (Diptera: Calliphoridae). J Forensic Sci. 2009;54(1):202-6.

15. Gabre RA, Adham FK, Chi H. Life table of Chrysomya megacephala (Fabricius) (Diptera : Calliphoridae). Acta Oecol. 2005;27(3):179-83.

16. Waite G. 4 Pests and Pollinators of Mango. Tropical Fruit Pests and Pollinators: Biology, Economic Importance, Natural Enemies, and Control. CABI. 2002;103.

17. Anderson D, Sedgley M, Short J, Allwood A. Insect pollination of mango in northern Australia. Crop Pasture Sci. 1982;33(3):541-8.

18. Wang $Z$, Wei $D$, Yang M, Dan J. The investigation of the varieties of mango insect pollinator in Guangxi and the preliminary observation on the habits and characteristics of the activities of such insects. Guangxi Agricultural Sciences (Chinese). 1999:2:4

19. Yang S, Liu Z. Pilot-scale biodegradation of swine manure via Chrysomyo megacephala (Fabricius) for biodiesel production. Appl Energy. 2014;113:385-91.

20. Li Z, Yang D, Huang M, Hu X, Shen J, Zhao Z, et al. Chrysomya megacephala (Fabricius) larvae: a new biodiesel resource. Appl Energy. 2012;94:349-54.

21. Song $C$, Yu H, Zhang M, Yang Y, Zhang G. Physicochemical properties and antioxidant activity of chitosan from the blowfly Chrysomya megacephala larvae. Int J Biol Macromol. 2013;60:347-54.

22. Saurin H. Maggot-Bioconversion Research Program in Indonesia Concept of New Food Resources Results and Applications (Final Report). 2005-2011.

23. Zhang M, Yu H, Yang YY, Song C, Hu XJ, Zhang GR. Analysis of the Transcriptome of Blowfly Chrysomya megacephala (Fabricius) Larvae in Responses to Different Edible Oils. PloS one. 2013;8(5):e63168.

24. Leal WS. Odorant reception in insects: roles of receptors, binding proteins, and degrading enzymes. Annu Rev Entomol. 2013;58:373-91.

25. Hansson BS, Stensmyr MC. Evolution of insect olfaction. Neuron. 2011;72(5):698-711.

26. Campobasso CP, Di VG, Introna F. Factors affecting decomposition and Diptera colonization. Forensic Sci Int. 2001;120(1-2):18-27.

27. Raju AS, Ezradanam V. Pollination ecology and fruiting behaviour in a monoecious species Jatropha curcas L.(Euphorbiaceae). Curr Sci. 2002;83(11):1395-7.
28. Grabherr MG, Haas BJ, Yassour M, Levin JZ, Thompson DA, Amit I, et al Full-length transcriptome assembly from RNA-Seg data without a reference genome. Nat Biotechnol. 2011;29(7):644-U130.

29. Haas BJ, Papanicolaou A, Yassour M, Grabherr M, Blood PD, Bowden J, et al. De novo transcript sequence reconstruction from RNA-seq using the Trinity platform for reference generation and analysis. Nat Protoc. 2013;8(8):1494-512.

30. Storey JD. The positive false discovery rate: a Bayesian interpretation and the q-value. Ann Stat. 2003;31(6):2013-35.

31. Zhang M, Yu H, Yang Y, Song C, Hu X, Zhang G. Analysis of the Transcriptome of Blowfly Chrysomya megacephala (Fabricius) larvae in responses to different edible oils. PLOS ONE. 2013:8(5):e63168.

32. Ache BW, Young JM. Olfaction: diverse species, conserved principles. Neuron. 2005:48(3):417-30

33. Arya GH, Weber AL, Wang P, Magwire MM, Negron YLS, Mackay TFC, et al. Natural variation, functional pleiotropy and transcriptional contexts of odorant binding protein genes in Drosophila melanogaster. Genetics. 2010;186(4):1475-U1623.

34. Libert S, Zwiener J, Chu X, VanVoorhies W, Roman G, Pletcher SD. Regulation of Drosophila life span by olfaction and food-derived odors. Science. 2007;315(5815):1133-7.

35. Robinson RAS, Kellie JF, Kaufman TC, Clemmer DE. Insights into aging through measurements of the Drosophila proteome as a function of temperature. Mech Ageing Dev. 2010;131(9):584-90.

36. Anholt RRH, Williams TI. The soluble proteome of the Drosophila antenna. Chem Senses. 2010;35(1):21-30

37. Swarup S, Williams TI, Anholt RRH. Functional dissection of Odorant binding protein genes in Drosophila melanogaster. Genes Brain Behav. 2011;10(6):648-57.

38. Graham LA, Davies PL. The odorant-binding proteins of Drosophila melanogaster: annotation and characterization of a divergent gene family. Gene. 2002;292(1-2):43-55.

39. Li B, Dewey CN. RSEM: accurate transcript quantification from RNA-Seq data with or without a reference genome. BMC Bioinformatics. 2011;12:323.

40. Anholt RRH, Dilda CL, Chang S, Fanara JJ, Kulkarni NH, Ganguly I, et al. The genetic architecture of odor-guided behavior in Drosophila: epistasis and the transcriptome. Nat Genet. 2003;35(2):180-4.

41. Zhou S, Stone EA, Mackay TFC, Anholt RRH. Plasticity of the Chemoreceptor Repertoire in Drosophila melanogaster. Plos Genetics. 2009;5(10):e1000681.

42. Carney GE. A rapid genome-wide response to Drosophila melanogaster social interactions. BMC Genomics. 2007;8.

43. Fujikawa K, Takahashi A, Nishimura A, Itoh M, Takano-Shimizu T, Ozaki M. Characteristics of genes up-regulated and down-regulated after $24 \mathrm{~h}$ starvation in the head of Drosophila. Gene. 2009:446(1):11-7.

44. Dalton JE, Kacheria TS, Knott SRV, Lebo MS, Nishitani A, Sanders LE, et al. Dynamic, mating-induced gene expression changes in female head and brain tissues of Drosophila melanogaster. BMC Genomics. 2010;11.

45. Fujii $\mathrm{S}$, Amrein $\mathrm{H}$. Genes expressed in the Drosophila head reveal a role for fat cells in sex-specific physiology. EMBO J. 2002;21(20):5353-63.

46. Swarup S, Morozova TV, Sridhar S, Nokes M, Anholt RRH. Modulation of feeding behavior by odorant-binding proteins in Drosophila melanogaster. Chem Senses. 2014;39(2):125-32.

47. Hallem EA, Carlson JR. Coding of odors by a receptor repertoire. Cell. 2006;125(1):143-60.

48. Hallem EA, Ho MG, Carlson JR. The molecular basis of odor coding in the Drosophila antenna. Cell. 2004;117(7):965-79.

49. Wang $P$, Zhang N, Zhou LL, Si S-Y, Lei CL, Ai H, et al. Antennal and behavioral responses of female Maruca vitrata to the floral volatiles of Vigna unguiculata and Lablab purpureus. Entomol Exp Appl. 2014;152(3):248-57.

50. DeGreeff LE, Furton KG. Collection and identification of human remains volatiles by non-contact, dynamic airflow sampling and SPME-GC/MS using various sorbent materials. Anal Bioanal Chem. 2011;401(4):1295-307.

51. Statheropoulos M, Spiliopouiou C, Agapiou A. A study of volatile organic compounds evolved from the decaying human body. Forensic Sci Int. 2005;153(2-3):147-55. 